\title{
INTERNET Y LOS NUEVOS MODELOS AUDIOVISUALES
}

RESUMEN

La penetración de Internet ha permitido el incremento exponencial de la circulación de contenido, así como el acceso al mismo. Creadores y consumidores ahora hacen parte de un mismo rol, lo que ha permitido que se generen tensiones acerca del control sobre el flujo de los contenidos que circulan en la red. Este trabajo busca ahondar en las condiciones que han permitido la generación de nuevos modelos audiovisuales que representan una alternativa frente a los intereses comerciales de las grandes compañías en internet. Así como mostrar las tensiones que estos generan frente al tema de la propiedad intelectual sobre estos contenidos y, visibilizar las diferentes opciones que se han creado al respecto.

Palabras clave: Internet, audiovisual, derechos de autor, modelos alternativos.

\section{INTERNET AND NEW AUDIOVISUAL MODELS}

\section{ABSTRACT}

Internet has significantly increased circulation of content as well as access to the same. Nowadays, creators and consumers share the same role, which has generated tensions about control of available content in the World Wide Web. This paper seeks to delve into the conditions that have allowed the generation of new audiovisual models and how they represent an alternative to the commercial interests of large companies on the Internet. Likewise, the objective is to show the tension generated by these alternative models against the issue of intellectual property and visualize the different options that have been developed in regard of the situation.

Keywords: Internet, audiovisual, copyright, alternative models

\section{[ CAMILO CALDERÓN ACERO ]}

Comunicador Social-Periodista de la Universidad Externado de Colombia. Estudiante de la Maestría en Industrias Culturales, Universidad Nacional de Quilmes, Argentina. E-mail: ccalderon国cap.uvq.edu.ar

Recibido: 8 de abril de 2013

Aceptado: 3 de agosto de 2013

nternet ha sido visto como el facilitador de una mayor difusión de contenidos, que garantiza de esta manera una circulación acorde al espíritu de acceso abierto en el que se basa la Sociedad de la Información. Este espíritu invita no sólo a las empresas vincularse con la red sino a participantes de todo tipo.

Sin embargo, esta democratización en la circulación de contenidos ha sido tildada en otros sectores como una conducta reprochable, de la mano de las denuncias de los empresarios sobre infracción al derecho de autor, las cuales han hecho eco en los Estados a través de legislaciones. Son numerosos los casos de propietarios de sitios web de descarga como Taringa, Cuevana o Megaupload, cuyos dueños han terminado en pleitos judiciales y en este último caso en la cárcel bajo cargos penales, cuya imputación viene de las grandes compañías productoras como Warner, Disney, Fox o Universal.

Para Ariel Vercelli,

La mayoría de las corporaciones comerciales y las instituciones estatales y comunitarias vinculadas a la gestión del derecho de autor han significado estas nuevas capacidades como problemáticas, como fuentes de ilegalidad o, directamente, como "piratería". Esto ha llevado a profundas discusiones sobre las formas de regular la producción, circulación y comercialización de obras intelectuales de las industrias (Vercelli, 2009, pág 82).

Erróneamente, los Estados han deslegitimado las demandas de los usuarios y los consumidores aducen que las empresas desconocen la naturaleza del compartir en la red, la cual no es comercial.

Según García Canclini (2011),

[...] todos estos grupos empresariales están desesperados, han expresado explícitamente que se debe combatir la piratería porque las descargas libres de música, de libros y revistas en sitios de Internet erosionan su nego- 
cio. Pero plantean el aspecto más superficial del asunto y vemos que cuando logran convencer a los gobiernos para ejecutar leyes de censura y represión, algunas veces éstas fracasan inmediatamente, como si la interacción en las redes desbordara, en horas, lo logrado por las leyes y las policías al respecto (Canclini, 2011, párrafo 19).

Es así como la decisión política ha ido en contravía de lo que se debería promulgar como desarrollo en la sociedad actual. Como postula el teórico Lawrence Lessig,

En una sociedad libre, con un mercado libre, apoyado por la libre empresa y el libre comercio, el papel del gobierno no es apoyar una forma de hacer negocio frente a las demás. Su papel no es escoger a los ganadores y protegerlos contra las pérdidas. [...] Un mundo en el que los competidores con ideas nuevas deben luchar no sólo con el mercado sino también con el gobierno es un mundo en el que los competidores con ideas nuevas no tendrán éxito. Es un mundo estático y de estancamiento cada vez más concentrado (Lessig, 2005, pág. 149)

Lo que esto permite dilucidar, es que las grandes compañías no buscan preservar el derecho de autor sino impedir que a este terreno lleguen competidores pequeños, para conservar así un monopolio de distribución.

Por esto, es necesario pensar una tercera vía que reconozca las verdaderas posibilidades de esta nueva realidad digital. Un nuevo modelo de gestión que en realidad permita erradicar esta tensión. Para Lessig,

[...] sólo porque un grupo con intereses específicos le pida apoyo al gobierno no se justifica que se conceda ese apoyo. Y sólo porque la tecnología haya debilitado una determinada forma de hacer negocio no se justifica que el gobierno deba intervenir para apoyar la forma antigua de hacer negocio (Lessig, 2005, pág. 148).

García Canclini plantea el reto de la siguiente manera, ¿Cómo ejercer plenamente tus derechos como ciudadano en la sociedad contemporánea si no cuentas con recursos tecnológicos y simbólicos para participar de las redes informáticas? Esto es lo que en definitiva forja ciudadanía, tal como acota Bustamante: "Estos flujos permean la sociedad deben ser tan libres, simétricos, abiertos, accesibles y veraces una democracia participativa hace falta garantizar los flujos de información a partir de los cuales se generan como sea posible" (Bustamante, 2010, pág. 2).

Esto se logra en la medida que se sobrepase el tema del Copyright para reconocer la importancia de la circulación de contenidos, entre ellos los audiovisuales, para la construcción de ciudadanía.

El conocimiento entendido como bien común es también un requisito indispensable para el desarrollo de una cultura plenamente creativa. No está limitada al ideal romántico de la originalidad exclusiva, pues se genera a partir de un enorme número de microcontribuciones, se distribuye por la idea de la recombinación, del remezclado, de la fusión, de la derivación, de la eliminación de todas las trabas a la creación, de la obra continua, ilimitada, fundamentalmente abierta. Maneja y transforma bajo las mismas reglas la novedad y la reconfiguración. Cultiva la colaboración, la construcción comunal del conocimiento y el compartir, tal como lo enuncia el ideal científico. La ciencia no habría avanzado si no hubiera sido ella misma comunal, abierta, acumulativa y recombinatoria- (Bustamante, 2010, pág. 3).

Esto se ha traducido en que "también las formas de gestión se volvieron más colaborativas, abiertas, menos jerárquicas y acompañaron la producción colaborativa y entre pares que caracterizan Internet (Stallman, 2002; Benkler, 2006). Por el contrario, otras formas de gestión vinculadas a la tradición de las industrias culturales de las corporaciones comerciales pasaron a ser más restrictivas, afectaron el acceso a la cultura, los derechos de copia, la libertad de expresión, la creatividad, la innovación y el carácter común de los bienes intelectuales (Vercelli, 2004, 2008; Lessig, 2006). Así, "los derechos de autor y los derechos de copia en la era digital pueden describirse como atravesando una situación dilemática: compartir o restringir, incluir o excluir" (Vercelli, 2009, pág. 94).

Para Lessig sólo es posible pensar en el éxito de una cultura digital, si ésta se equipara con la libertad.

Una cultura libre apoya y protege a creadores e innovadores. Esto lo hace directamente concediendo derechos de propiedad intelectual. Pero lo hace también indirectamente limitando el alcance de estos derechos, para garantizar que los creadores e innovadores que vengan más tarde sean tan libres como sea posible del control del pasado. Una cultura libre no es una cultura sin propiedad, del mismo modo que el libre mercado no es un mercado en el que todo es libre y gratuito. Lo opuesto a una cultura libre es una "cultura del permiso"--una cultura en la cual los creadores logran crear solamente con el permiso de los poderosos, o de los creadores del pasado (Lessig, 2005, pág. 8).

Abrir este abanico sólo es posible a partir de la circulación de contenido. La reivindicación de la copia como mecanismo de actuación en una sociedad que valida el compartir información debe sobreponerse al concepto que iguala tal práctica con la piratería.

En el caso de la libre distribución audiovisual no sólo se trata de la transmisión de una obra sino de la percepción de 
mundo sobre un asunto, sobre una cultura. Se acercan y se ponen a dialogar visiones de una manera cercana para los ciudadanos. Éstas son las posibilidades que eventualmente deben ser consideradas en un debate, mucho más que las de derechos de autor, que erróneamente se ha creído que se afecta.

Como manifiesta Joost Smiers, hay que comprender este tema dentro el marco de sus posibilidades para la diversidad cultural.

Muchos países no tienen la capacidad de permitir que sus artistas creen trabajos audiovisuales de calidad que puedan competir con los productos extranjeros provenientes de las grandes industrias culturales. Esos países tendrían que acceder a unos medios económicos que les permitieran construir infraestructuras de producción y distribución al tiempo que dieran a los artistas la posibilidad de desarrollar su talento (Smiers, 2006, pág. 257).

Cuando se reduce el tema de la descarga de contenidos a un asunto puramente de regalías por propiedad intelectual sale a flote un sesgo mercantilista que limita las posibilidades de reconvertir esta práctica hacia su verdadero sentido. Esto para las compañías no es fácil de asimilar. Como indica García Canclini,

"Lo que el juego actual nos está enseñando es que, por un lado, todavía hay grandes poderes en la industria cultural y comunicacional muy concentrados, con capacidad de ahogar a los pequeños; por otro, que los grupos alternativos y las redes o flujos perseguidos o atacados parcialmente por las industrias empresariales también tienen dificultades para sobrevivir" (Canclini, 2011, párrafo 20).

Es allí donde urge la presencia del Estado, para asegurar que el acceso, disfrute y regalías de esta nueva cultura digital audiovisual sea plural y diversa.

Es el deber de los legisladores, en otras palabras, asegurar que los cambios que ellos crean, en respuesta a las peticiones de aquellos perjudicados por una tecnología que cambia, son cambios que preservan los incentivos y oportunidades para la innovación y el cambio (Lessig, 2005, pág. 49).

\section{Como subraya Smiers}

Está claro que todos los objetivos sociales y culturales de las demás reglamentaciones a favor de la diversidad cultural, que recurren a mecanismos tales como cupos, subsidios y sistemas impositivos, entre otros, no podrían cumplirse si los espectadores u oyentes no tienen acceso al contenido (Smiers. 2006, pág. 263).
Por lo que es necesario repensar una regulación que permita y no restringa la circulación de contenido en la red.

Ahora sólo falta que los Estados diseñen políticas para hacer valer este potencial de Internet.

Las redes son magníficas, dan posibilidades inexploradas hasta ahora, pero también generan conflictos nuevos y obligan a repensar interrogantes como los planteados aquí: qué es la propiedad intelectual, qué es lo mío, qué es lo de la comunidad, qué es lo público, qué es lo que necesitamos compartir para ser sociedad (García Canclini, 2011, párrafo 35).

\section{EL ACCESO A UN DERECHO}

Mientras los Estados aún buscan cómo hacerle frente a este nuevo escenario, aparecen cada vez más casos en el mundo que evidencian una nueva concepción del modelo de negocio del audiovisual en internet ¿Qué opción les queda a los creadores en países donde aún la ley está estrechamente ligada a una concepción rígida de la propiedad intelectual? Lessig plantea el reto de esta forma:

¿Hay otra forma de asegurar que se pague a los artistas sin convertir en criminales a 43 millones de estadounidenses? ¿Tiene sentido si hay otras maneras de asegurar que se pague a los artistas sin convertir a los EE.UU. en un país de criminales? (Lessig, 2005, pág. 227).

Por eso, pensar en nuevos modelos de gestión implica considerar que existen intereses distintos al comercial en internet. Como acota Vercelli, "las nuevas capacidades en manos de los usuarios-finales fue acompañada por una creciente colaboración y asociación entre todo tipo de autores y productores no industriales de obras” (2009, pág. 82).

Se abre así la posibilidad de pensar otro tipo de modelos que den sustento a esas perspectivas.

No sólo existe la economía comercial, donde la medida del acceso está en la simple métrica del precio. Existe también una economía del compartir, donde el acceso a la cultura no es regulado por el precio, pero sí por un complejo conjunto de relaciones sociales. Esas relaciones no son simples. Y de hecho, esas relaciones resultan insultadas con la simplicidad del precio ${ }^{1}$ (Lessig, 2008, pág. 145).

1 Traducción propia del original en inglés: "There exists not just the commercial economy, which meters access on the simple metric of price, but also a sharing economy, where access to culture is regulated not by price, but by a complex set of social relations. These social relations are not simple. Indeed, these relations are insulted by the simplicity of price" 
Esta "economía del compartir" o "consumo colaborativo" 2 no sólo es factible, sino que y existen modelos que funcionan de esta forma y en internet este tipo de iniciativas han encontrado un lugar para florecer. Algunas posiciones sin embargo consideran que este modelo riñe con el comercial, pero en la propuesta de Lessig se contempla una coexistencia que se materializa en una "economía hibrida". Para él, en este caso, "lo comercial y la economía del compartir coexisten. De hecho, se complementan entre ellas $[. .$.$] Entendemos que cosas similares pueden ser$ ofrecidas dentro de diferentes economías. Celebramos esa diversidad”3 (Lessig, 2008, pág. 150).

Sin embargo, lograr esta coexistencia genera tensiones propias de quienes se aferran al primer modelo. Estas tensiones surgen "principalmente, en las capacidades diferenciales de gestión de los diferentes grupos sociales" (Bijker, 1995 citado por Vercelli, 2009, pág. 93), pues los representantes de estos modelos basados en el compartir se encuentran en el trayecto con factores que obstaculizan el desarrollo de sus propuestas. Esto significa presiones tanto tecnológicas como sociales y políticas. tivas, pues considera que las alternativas que hasta ahora se han dado tienen bajo empoderamiento.

Este es el caso de licencias creativas o abiertas (Creative Commons) "que le permiten a los creadores escoger selectivamente qué derechos se reservan y qué derechos liberan sobre sus obras" (Vercelli, 2009, pág. 96), al brindar una alternativa al sistema de copyright. Su implementación por el momento es la que más ha logrado aceptación social como técnica, pero aún así, es un mecanismo que resulta insuficiente en temas como "lograr que los artistas de distintas partes del mundo, así como sus productores y patrocinadores, obtengan beneficios económicos" o "que sólo abarcan a los artistas que deciden adherirse a su filosofía. Y es obvio que los grupos de empresas culturales, que tienen la propiedad de gran parte de nuestro legado cultural del pasado y el presente, no elegirán esa opción” (Smiers, 2006, pág. 288).

Este tipo de elementos plantean que el tema de nuevos modelos de gestión para los audiovisuales en internet no se puede abarcar de forma totalizante, a través de una solución hegemónica para toda la cantidad de perspectivas, pues

Es decir, las formas de gestión de derechos en los entornos digitales también dependen de las tecnologías digitales disponibles, de la arquitectura política de Internet $y$, sobre todo, de las formas de producción de obras intelectuales que tengan los usuarios finales de redes

distribuidas como Internet (Vercelli, 2009, pág. 94).

Para Lessig, lograr puntos de acercamiento no es tan disparatado, pues la existencia de un modelo no necesariamente perjudica al otro. Esta cuestión también plantea la necesidad de que estos modelos sean legitimados, pues había instituciones estatales y comunitarias que gestionaban el derecho de autor en la era pre Internet (Vercelli, 2009:96).

Smiers lo señala de esta forma, "Esa es la combinación deseable. Cuando el mercado no ofrece esa diversidad, las autoridades tienen el deber de apoyar lo nuevo, lo más débil y lo que supone una contribución significativa a la diversidad” (2006, pág. 273). Su posición hace más énfasis en el rol que le corresponde al Estado en el apoyo de estas inicia-

2 Término original Economy Sharing en ingles, acuñado por Lawrence Lessig.

3 Traducción propia del original en inglés: “ Commercial and sharing economies coexist. Indeed, they complement each other. (...) We all understand that similar things can be offered within different economies. We celebrate this diversity".

algunas cuentan con un enfoque más técnico, comercial o ideológico. Es por eso, que puede que un modelo basado en Creative Commons sirva para algunos casos y para otros no sea la mejor opción, y allí es donde cabe pensar más a fondo una economía híbrida.

Por ejemplo, Smiers plantea el potencial de las Creative Commons al decir que esta "licencia es adecuada para museos y archivos que quieran difundir su legado cultural sin que nadie se apropie de él o lo use con fines inadecuados" (2006, pág. 288). Este es el caso del British Council que ha puesto en línea una serie de documentales realizados en la época de la Segunda Guerra Mundial y que habían sido almacenados por la Filmoteca Nacional Británica. “Tras un año de trabajo de restauración y digitalización, el Consejo Británico ha publicado en Internet estos documentales bajo licencia Creative Commons y, además, ofrece a los usuarios la posibilidad de descargarlos libremente" (Velasco, 2012, párrafo 1).

La idea, apoyada por Google, brinda la posibilidad de acceso a 80 documentales clásicos que muestran el estilo de vida británico de los años 40 a través de la web del Consejo 
Británico (http://film.britishcouncil.org/british-councilfilm-collection) y en el canal de Vimeo de la institución.

El hecho de que empresas comerciales se vinculen a este tipo de iniciativas implica por un lado una oportunidad, pero también el riesgo de que este canal de acceso vuelva a quedar cerrado por su injerencia. La solución no está en cerrar este camino sino en permitir que además de existir la opción más "comercial" se permita acceder, modificar y apropiarse del contenido. Para que esta coexistencia se pueda dar es necesaria la intervención estatal, de no ser así esos mismos modelos alternativos serán cooptados por las grandes compañías, al desplazar de nuevo al Estado, quien llega de último al nuevo escenario que plantea la distribución de contenidos en internet.

Por ejemplo, en el 2012 el Instituto Mexicano de Cinematografía (IMCINE) llegó a un acuerdo con el administrador de contenidos YouTube, con el objetivo de distribuir en su plataforma 71 títulos de cortometrajes mexicanos y posteriormente largometrajes. Su presidenta, Marina Stavenhagen, manifestó que el énfasis debe estar en la red. "El futuro del cine mexicano está en la digitalización, Internet y las redes sociales. Vamos a ver cómo funciona, pensamos que es una alternativa de salida interesante y de impacto 2011, párrafo 3), recalcó en una entrevista para un portal sobre las audiencias, y vamos a ver cómo nos va" (Badillo, de noticias de su país.

buida de los diferentes grupos sociales la que construirá el funcionamiento o no funcionamiento de estas soluciones [artefactos, plataformas o modelos de gestión]” (Vercelli, 2009, pág. 100).

Es por eso que la motivación ideológica, sea o no comercial, no riñe con el espíritu del modelo alternativo. Pues más allá de la forma de accionar consciente o inconsciente, la utilidad de estos modelos reside en los efectos que logran y en lo que todos ellos coinciden es que crean comunidad, al generar participación y al visibilizar la diversidad.

\section{CÓMO ACTÚAN LOS MODELOS ALTERNATIVOS}

Dentro de estos modelos de economía híbrida en los cuales se insertan características del consumo colaborativo, Lessig habla de modelos de economías del compartir delgadas (thin sharing economies) y economías del compartir gruesas (thick sharing economies). Las primeras son "aquellas economías en las que la motivación es principalmente hacia uno mismo. En las economías del compartir gruesas las motivaciones son menos ambiguas entre la motivación dirigida hacia uno mismo y la dirigida hacia los otros. Así, en las economías del compartir delgadas, la gente no basa el intercambio en el precio o dinero. "Pero hacen este intercambio simplemente porque los hace estar mejor, o porque estos son un subproducto inevitable de algo que de todos modos querían hacer por razones que tienen que ver puramente con ellos mismos. A una persona no tiene por qué interesarle necesariamente que sus acciones puedan ayudar a alguien más. No hay voluntad independiente para ayudar a otra persona. La motivación es sobre ella misma ${ }^{4}$ (Lessig; 2008, pág. 152).

Lessig en su caracterización de

Esta alianza, si bien es cierto permitirá una mayor circulación de contenido, muestra el creciente poder que han empezado a tomar compañías como YouTube, al crear otra forma de poder hegemónico dentro de las redes. ¿Podría ser de otra forma? ¿Tiene menos valor este modelo que el desarrollado a través de las Creative Commons?

Es importante que existan ya que su permanencia o no, no se va a definir por imposición sino en el plano de la apropiación social. Como manifiesta Vercelli,

El éxito o fracaso de las soluciones propuestas por los grupos sociales no responde a aquello que está expresado en las regulaciones o a propiedades intrínsecas de los mismos artefactos [...] Será la acción política distri- estos modelos delgados excluye el factor monetario pero es más preciso al expresar que su rol no es protagónico y que en los modelos híbridos esa importancia es la que puede

4 Traducción propia del original en inglés: "those economies where the motivation is primarily me-regarding; "thick sharing economies" are economies where the motivations are at least ambiguous between me and thee motivation. Thus, in thin sharing economies, people do not base an exchange on price or money. But they're making this exchange simply because it makes them better off, or because it is an unavoidable by- product of something they otherwise want to do for purely me- regarding reasons. One person doesn't necessarily mind that his actions might be helping someone else. But there's no independent desire to help someone else. The motivation is about me". 
establecer una graduación desde los modelos delgados a los gruesos. "Entre más pensemos que lo que hay es una motivación hacia los otros, más gruesa será la comunidad. La distinción entre gruesa y delgada será importante cuando se consideren las diferencias entre las económicas del compartir"s (Lessig, 2008, pág. 154).

El factor económico incide pero no por eso el modelo es un modelo comercial. Por las características del material audiovisual en el cual se basan algunos modelos, se podría hablar de modelos cuya base es delgada y otros, cuya base es más amplia o gruesa. Puede que el valor de los primeros para lograr un acceso equitativo en la red sea mínimo y que a sus creadores no les interese ampliar el modelo de gestión, pero aún son estrategias que se conforman fuera de las lógicas de las grandes empresas y que a su modo representan intereses independientes.

Es por eso que Lessig (2008) establece que estos modelos híbridos, según el grado de gestión, construyen espacios de comunidad (Community Spaces), espacios colaborativos (Colaborative Spaces) y comunidades (Community) propiamente dichas, cuyos modos de gestión funcionan siempre distinto, incluso dentro de casos de la misma categoría ${ }^{6}$.

Se podría pensar en un primer nivel, si se estableciera un camino desde los modelos delgados a los gruesos, éste sería el caso de los espacios de comunidad. La forma más clásica de este tipo de estrategia son los catálogos online de películas, sin importar si son pagos como Vesvi ${ }^{7}$ (Argentina) o gratuitos como Cuevana (Argentina). Ahí todos quienes participan en ellos e incluso sus creadores no buscan conscientemente un objetivo profundo al brindar la posibilidad de ver audiovisuales en internet. Se abre un espacio, pero esa apertura no cohesiona de forma tal que sus integrantes puedan elevar acciones más profundas que simplemente tener una afinidad de gustos por un tipo de producto audiovisual.

Otro ejemplo de este tipo Indyon.tv, un portal colombiano que se creó en septiembre de 2012 y cuyo objetivo es visibilizar las películas independientes en Colombia y que permite ver productos audiovisuales gratuitos y pagos con un costo de uno a cinco euros. Más allá de la ventana que

5 Traducción propia del original en inglés: "and that the more we think that there is a thee- motivation, the thicker the community is. This distinction between thick and thin will be important when considering differences among sharing economies".

6 Lessig destaca en esta división algunos ejemplos .Para verlos con más detalle, dirigirse al texto Remix Making art and commerce thrive in the hybrid economy.

7 En el recuadro final se puede consultar la dirección web de este portal y de los siguientes que se mencionan. ofrece para los productores, no se contempla la visibilización de los integrantes que acuden al sitio, a pesar de ser necesario un registro para poder ver el material. Esta falencia no deslegitima su accionar, puesto que su modelo tiene el germen la decisión de ofrecer un contenido alterno. No por nada sus creadores contemplan un manifiesto en el cual se indica que "es la independencia, la autonomía, la libertad, la que impulsa el ansia de conocimiento, ese que genera reflexión y conciencia" ${ }^{8}$. Esto significa, que con ajustes a la acción política que inspira, se puede materializar este propósito o simplemente quedar en una declaración de buenos deseos que no cohesione, debido a las mismas presiones del mercado.

El caso del proyecto Cinépata en Chile, impulsado por el escritor y cineasta Alberto Fuguet es un buen ejemplo de lo que sería un espacio colaborativo. En este portal web se ofrece de manera gratuita un catálogo cine latinoamericano, documental, cortometraje y hasta música. Del mismo que en Indyon.tv hay un manifiesto de sus creadores, pero la diferencia es que sus postulados se materializan en el mismo funcionamiento del portal.

Como se indica en su manifiesto: "Acá en Cinépata queremos hacer una suerte de sala de cine-arte o cine-alternativo virtual, donde puedas encontrar películas y cortos que muchas veces nacen o mueren en festivales, o que apenas pasan por una pantalla sucia y deshilachada de una ciudad grande. Cinépata quiere también ser una comunidad de cinéfilos que se intercambian información y que confían en sus gustos" $"$

Como apunta Lessig, "aquellos quienes participan en un espacio colaborativo piensan que su trabajo es diferente. O más acertadamente, al menos algunos (una cantidad significante) creen que están construyendo algo juntos"10 (2008, pág. 196). Es por esta razón que Cinépata usa Creative Commons como mecanismo de licencia para circular el material en el sitio, al reafirmar que en ese espacio el tema del copyright es entendido de otra forma.

Dado que en Cinépata no es necesaria la suscripción, no es posible de constituir la comunidad, aunque los creadores sí tengan claro que llevar su proyecto a ese lugar implica objetivos diferentes que enviarlo a otros. Esta característica del modelo también ha permitido el desarrollo de una particularidad:

$\mathbf{8}$ Ver manifiesto: http://indyon.tv/portal/manifiesto

9 Ver manifiesto: http://www.cinepata.com/articulos/2010/10/ahora-aqui-en-tu-pantalla-favorita-gratis/

10 Traducción propia del original en inglés. Those participating in a collaboration space think their work is different. Or more accurately, at least some (significant portion) of those on a collaboration space believe they are there to build something together". 
no es vital la generación de lucro. Es por esta razón, que uno puede encontrar para su funcionamiento socios (Partners) y no inversores, puesto que un partner establece una afinidad ideológica con el proyecto más allá de lo comercial.

En este punto, volviendo a cómo las compañías comerciales y el Estado se pueden vincular a estos modelos, es posible que haya un punto de unión en su participación en este tipo de proyectos. En el caso de Cinépata por ejemplo, dentro de sus partners está una marca de ropa, una revista y la biblioteca de la Universidad Diego Portales. Así mismo, en el portal se publica material escrito de análisis como columnas y hay cubrimientos especiales de festivales como es el caso del Festival de Cine de Valdivia, donde algunos cortometrajes se presentaron en el portal.

Cuando se construye un vínculo mayor entre los participantes, se construye finalmente comunidad, lo que equivaldría a un modelo grueso de economía del compartir. Esto requiere esfuerzos mayores y establece un espacio donde "todos ellos crean una clase de valor mayor, que es mayor al que el creador obtiene al compartir. Y como en cualquier comunidad, cuando más gente contribuye, y observa la contribución de los otros, todos se sienten más ricos"11 (Lessig, 2008, pág. 217). Éste es el caso del documental argentino La Educación Prohibida y el portal Comunidad Zoom. Los dos, sin ser simple coincidencia basan su modelo en la instauración de Creatives Commons para la circulación del material. Este mecanismo, por ahora es el que más posibilidades brinda en internet para el acceso de obras audiovisuales.

La Educación Prohibida fue pensada desde su temática como la visibilización de otra forma de entender los procesos educativos, por lo cual las licencias abiertas eran compatibles dentro de su estrategia de difusión en internet. El uso de Creative Commons sólo fue una de las herramientas libres que se usaron para su realización, pues también hubo uso de software libre ${ }^{12}$.

Con respecto a los casos de creación de comunidad, la iniciativa de Comunidad Zoom en Argentina resulta pertinente porque apunta a agrupar características antes mencionadas. Funciona igualmente ofreciendo un catálogo de películas, en su mayoría de forma gratuita, cuyo énfasis es el cine arte. Para ajustarse en el tema legal ha buscado repositorios mundiales donde se conserva ya material de dominio público con lo cual es posible ver un buen número

11 Traducción propia del original en ingles: "They all create a kind of value that more than the creator gets to share. And as with any community, the more people contribute, and see others contribute, the richer everyone feels".

12 Ver en detalle: http://www.educacionprohibida.com/distribucion/aspectos-legales/ de clásicos del cine. Igualmente hay películas, cortometrajes y documentales que ya han subido material con licencia Copyleft (Creative Commons) y el portal simplemente agrupa estas iniciativas.

Los usuarios en este caso cumplen un rol mayor y por eso desde su mismo nombre se enfatiza el sentido de comunidad que tiene el portal (más de 60 mil usuarios). Además de invitar a subir material, se les impulsa a los usuarios a crear un perfil, comentar las películas, realizar críticas y traducir los subtítulos. De esta forma se construye una relación con el usuario, que al final puede facilitar procesos como la donación de fondos para el proyecto. Ésta es una de las modalidades de recaudo, pues también se pueden comprar espacios de publicidad.

\section{LO QUE LAS CREATIVE COMMONS NO SOLUCIONAN}

Otra perspectiva plantea que estos modelos que incluyen Creatives Commons "no desarrollan un modelo económico sobre cómo se pueden ganar la vida los artistas en estos nuevos países de las maravillas" (Smiers, 2008, pág. $116)$ y por lo tanto su gestión como modelos alternativos es de poca utilidad. Esta postura ataca frontalmente la sustentabilidad de los modelos híbridos y sustenta que la mejor forma para resolver el problema es reforzar el rol del Estado para reparar la desigualdad y lograr diversidad. Esto puede ser mediante una reinterpretación de la regulación en temas de propiedad y contenido (Smiers, 2006, pág. 247).

Una de las manifestaciones que sirven para ese propósito es que el Estado intervenga con algún tipo de subvención o creación de redes audiovisuales que apoyen los productos que no puedan por sí solos resistir la competencia del mercado y con eso se asegure recuperar los ingresos y así mismo, que este tema no influya en su realización. "El neoliberalismo abstracto y el libre comercio deberían dejar de ser los únicos principios rectores de la distribución de películas en las distintas regiones del mundo" (Smiers, 2006, pág. 247).

En Latinoamérica, al seguir un poco esta línea se abrió la posibilidad de una plataforma latinoamericana de distribución audiovisual, la cual incluye un portal de internet para este fin. El Banco Interamericano de Desarrollo destinó recursos por primera vez en su historia, para una propuesta de este tipo, que fue presentada por las autoridades relacionadas con cultura de México, Ecuador, Perú, Bolivia, Uruguay y Colombia ${ }^{13}$.

13 Ver más información sobre el acuerdo: http://www. mincultura.gov.co/?idcategoria $=49220$ 
La injerencia estatal debe ser el apoyo mas no el lugar desde el cual se generen estos espacios, pues la virtud de ser bienes comunes reside justo en ese empoderamiento que le pertenece a todos y delegar esa gestión en el Estado puede terminar igualmente, concentrando temáticas y favoreciendo posturas.

Al pensar de esta forma todos los modelos antes mencionados, estos cumplen con la función de distribuir un material y serían competencia entre sí. Pero si se amplia la perspectiva, lo que los hace funcionales no es el producto que ofrecen en sí, como manifiesta Lessig cuando habla de porqué el software libre tuvo éxito a pesar de no estar anclado en un sistema comercial como sus competidores: "la era vender beneficios y no características"14(2006:182). Esta vuelta de mirada al tema rescata el verdadero rol que cumple la red en

14 Traducción propia del original: "The key was to sell "benefits" and not "features." la vida de quienes las usan. "En este sentido, Internet es más un medio de producción de obras intelectuales colaborativo, distribuido y entre pares que un canal centralizado de distribución y comercialización en manos de las industrias culturales" (Vercelli, 2009, pág. 130).

A este objetivo de descentralización es al que apuntan los modelos antes mencionados, algunos con un mayor grado de impacto socio-político en su gestión y otros con menor grado de empoderamiento, pero que coinciden en que ponen de manifiesto las demandas sociales que el modelo de economía comercial no soluciona y para el cual el sistema actual de derechos de autor y copyright no es la respuesta. Es necesario avanzar en el reconocimiento y legitimización de estas prácticas por parte del Estado. La visibilización surge entonces como primer paso para empezar a crear esos puentes que a la postre permitan mejores estrategias de abordaje para construir una alternativa fortalecida frente a la oferta guiada por el mercado.

\begin{tabular}{lll} 
Modelo de dffusión audiovisual en internet & Dirección electrónica & País \\
\hline Indyon.tv & http://indyon.tv/portal/ & Colombia \\
\hline Cinepata & http://www.cinepata.com/ & Chile \\
\hline Vesvi & http://www.vesvi.com/ & Argentina \\
\hline Cuevana & http://www.cuevana.tv/ & Argentina \\
\hline Comunidad Zoom & http://comunidadzoom.com/ & Argentina \\
\hline La Educación Prohibida & http://www.educacionprohibida.com/ & Argentina \\
\hline
\end{tabular}

\section{BIBLIOGRAFÍA}

Badillo, J., M. (2011). En México confían en Internet para ganar público cinematográfico. Escribiendo cine. Recuperado de <http://www.escribiendocine.com/ noticias/en-mexico-confian-en-internet-para-ganarpublico-cinematografico>

Bustamante, D., J. (2010). “La cuarta generación de derechos humanos en las redes digitales" En: Revista Telos. No. 85. Cuadernos de Comunicación e Innovación. Recuperado de http://sociedadinformacion.fundacion. telefonica.com/seccion $=1268 \&$ idioma $=$ es ES\&id $=2010110411480001 \&$ activo $=6$.do

García, C., N. (2011). Cibernética, conocimiento y poder, entrevista de Diego Lizarazo. Revista Versión, Estudios de Comunicación, Política y Cultura. No. 27. Recuperado de http://version.xoc.uam.mx/index. php?option $=$ com_content $\&$ view $=$ article $\&$ id $=92$ : ciber netica-conocimiento-y-poder\&catid=38:versiones-deldialogo\&Itemid $=46$
Lessig, L. (2005). Cultura Libre. Cómo los grandes medios usan la tecnología y las leyes para encerrar la cultura y controlar la creatividad. Recuperado de http://www. worcel.com/archivos/6/Cultura_libre_Lessig.pdf (2008). Remix: Making Art and Commerce Thrive in the Hybrid Economy. Nueva York: Penguin Press. Recuperado de http://www. bloomsburyacademic.com/pdf\%20files/Remix.pdf

Smiers, J. (2006). Un mundo sin copyright. España: Editorial Gedisa. 384 p.

Smiers, J. y Van, S., M. (2008). Imagine... No copyright. Barcelona: Editorial Gedisa. 240 p.

Velasco, J. (2012). Alt 1040. Hipertextual. Recuperado de $<$ http://alt1040.com/2012/05/el-consejo-britanicocomparte-bajo-creative-commons-sus-documentales $>$.

Vercelli, A. (2009). Repensando los bienes intelectuales. Recuperado de www.arielvercelli.org/rlbic.pdf 